\title{
Pneumopericardium causing pericardial tamponade
}

\author{
Ryan Fink ${ }^{1}$ \\ ${ }^{1}$ Oregon Health \& Science University
}

May 13, 2020

\begin{abstract}
Pneumopericardium, or gas in the pericardial sac, is a rare condition that typically presents with chest pain or pericarditis, but can be severe enough to cause tamponade physiology. The images presented show the typical appearance of pneumopericardium. This patient had tamponade physiology requiring a pericardial drain in the operating room.
\end{abstract}

Title: Pneumopericardium causing pericardial tamponade

Author information: Ryan J Fink, M.D.

Oregon Health \& Science University

Corresponding author:

Ryan J. Fink, M.D. Oregon Health \& Science University Department of Anesthesiology and Perioperative Medicine 3181 SW Sam Jackson Park Blvd Mail Code UHS2 Portland, OR 972395034942075 finkr@ohsu.edu

Clinical Trial number: Not applicable

Prior Presentations: Not applicable

Acknowledgements: Not applicable

Word and Elements : Words: 249

Images: 2

Abbreviated title: Pneumopericardium tamponade

Summary statement: Not applicable

Funding statement: There was no separate funding provided; non-clinical time is supported solely from departmental sources.

Conflicts of interest: The author declares no competing interests.

Key words: Anesthesia, critical care medicine, cardiovascular disorders

Key Clinical Message: Pneumopericardium can be severe enough to cause pericardial tamponade physiology. These patients can be hemodynamically unstable and require pericardial drainage.

Case and Image Description

Pneumopericardium, or gas in the pericardial sac, is a rare condition that results from complications from thoracic trauma, malignancies, mechanical ventilation, or can be iatrogenic. ${ }^{1}$ It typically presents with chest 
pain, pericarditis, or even tamponade physiology. ${ }^{1}$ The patient in the images had a repeat Nissen fundoplication 6 months prior to presentation. He presented with shortness of breath, chest pain, hypotension, tachycardia, and a pulsus paradoxus of $20 \mathrm{mmHg}$, consistent with pericardial tamponade.

These images are typical of pneumopericardium. The chest radiograph (figure 1) shows a band of gas curving around the cardiac chambers, with a sharp demarcation by the pericardium (arrows). The computed tomography scan (figure 2) shows dependent air-fluid levels in the pericardial sac. It may be challenging to distinguish pneumopericardium from pneumomediastinum, but the latter usually presents as multiple streaks of radiolucency that are not confined to the cardiac chambers. ${ }^{2}$ Diagnosis of pneumopericardium, particularly if severe enough to cause pericardial tamponade, is especially important to diagnose preoperatively, as anesthetic induction can be life threatening. Point of care ultrasound was not attempted as the diagnosis of pneumopericardium was already made and air in the pericardial sac can make ultrasound imaging challenging, though not impossible.

This patient required emergent pericardial window. Air and gastric contents were found in the pericardial sac, and esophagogastroduodenoscopy confirmed the suspicion of a gastropericardial fistula, a complication from the surgical repair of a recurrent hiatal hernia. He underwent an esophagostomy diversion at that time, but eventually received an esophagogastric anastomosis for gastrointestinal tract continuity.

\section{Figure Legends}

Figure 1: Chest radiograph which shows a band of gas curving around the cardiac chambers, with a sharp demarcation by the pericardium (arrows).

Figure 2: Computed tomography scan which shows dependent air-fluid levels in the pericardial sac.

Author Contributions:

Author 1: Dr. Fink collected the images, obtained IRB approval, and wrote the text, and has no conflicts of interest, financial disclosures, and funding sources.

References:

1. Gołota JJ, Orłowski T, Iwanowicz K, Snarska J: Air tamponade of the heart. Kardiochir Torakochirurgia Pol 2016; 13: 150-3

2. Bejvan SM, Godwin JD: Pneumomediastinum: old signs and new signs. AJR Am J Roentgenol 1996; 166: $1041-8$ 

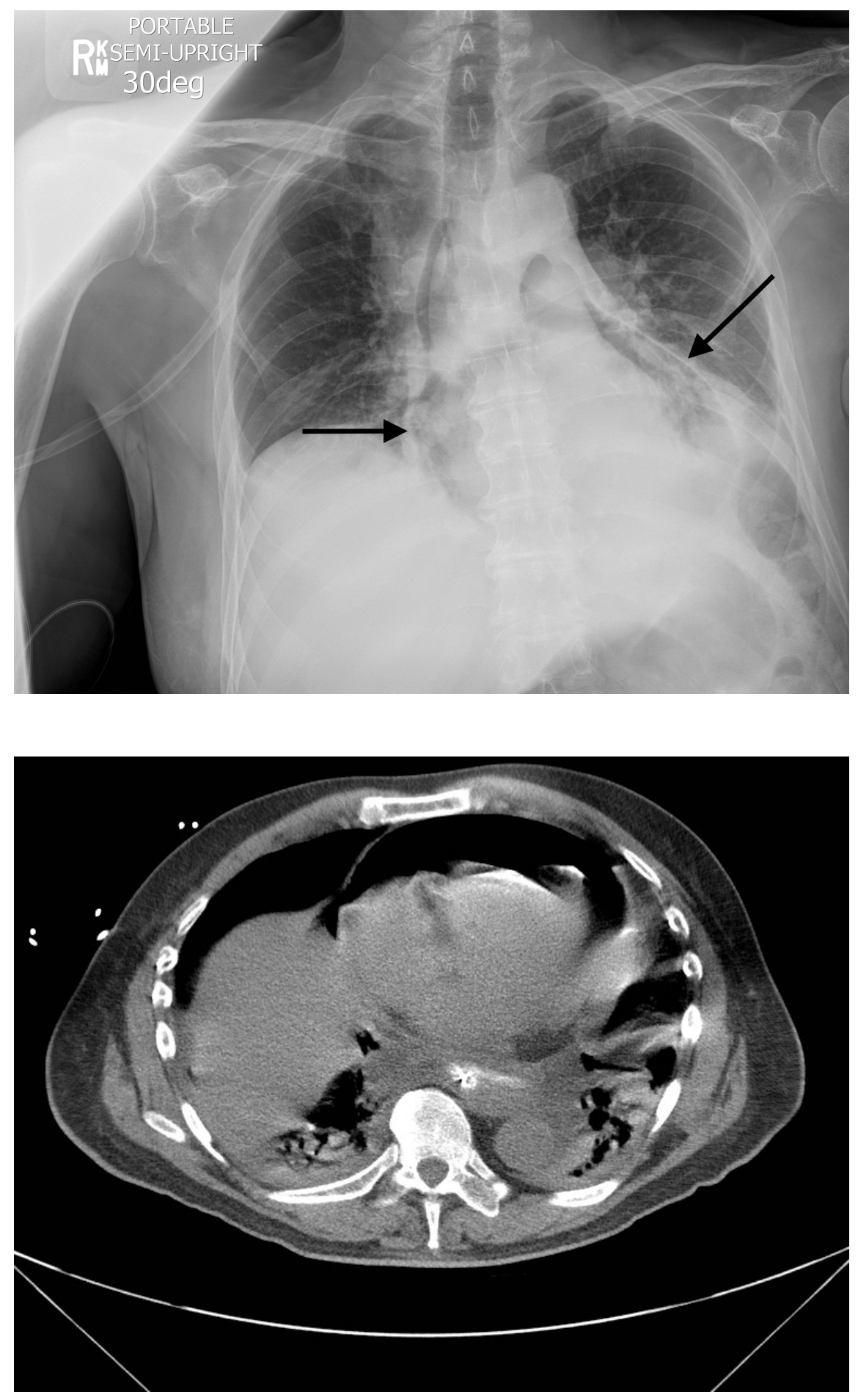